\title{
LA GESTIÓN DE PERSONAS EN EMPRESAS DEL SECTOR BIOTECNOLÓGICO FARMACÉUTICO\&
}

\section{THE PERSONS MANAGEMENT IN ENTERPRISES OF BIOTECHNICAL PHARMACIST SECTOR}

\author{
Yanela Cadalzo Díaz", María Julia Becerra Alonso ${ }^{2, *}$, Idania Caballero Torres ${ }^{3}$, \\ Mirelis Albojaire Santamaría ${ }^{4}$
}

\section{RESUMEN}

El objetivo de este artículo es establecer algunos requerimientos que demanda la gestión del capital humano en empresas de alta tecnología del sector biotecnológico farmacéutico cubano. El estudio se realiza a partir del empleo del método histórico lógico, la modelación, así como el análisis documental, la observación directa, y el método de expertos. Para cumplir con el objetivo general fue necesario utilizar el estudio de caso como método de investigación científica, que permite investigar la gestión del capital humano de las Empresas de Alta Tecnología del sector biotecnológico farmacéutico cubano, en su contexto real y con todas sus complejidades asociadas. Los resultados conducen al establecimiento de un modelo conceptual particular que tiene en cuenta las singularidades de un sector que se distingue por el uso intensivo del conocimiento y empresas de ciclo completo. Además, se muestra un procedimiento de aplicación general, con procedimientos específicos de trabajo, para cuyo diseño se tomaron en consideración las mejores prácticas desarrolladas en diferentes centros en materia de capital humano.

Palabras clave: Competencias, empresas de alta tecnología, gestión.

\section{ABSTRACT}

The objective of this article is to establish some requirements demanded by the management of human capital in high technology companies of the Cuban pharmaceutical biotechnology

*XIX Convención Científica de Ingeniería y Arquitectura, celebrada en La Habana, Cuba del 26 al 30 noviembre del 2018.

${ }^{1}$ Instituto Finlay de Vacunas. La Habana, Cuba. ycadalzo@finlay.edu.cu

${ }^{2}$ Universidad Tecnológica de La Habana José Antonio Echeverría, Facultad de Ingeniería Industrial. La Habana, Cuba. mjuliab@ind.cujae.edu.cu

${ }^{3}$ Centro de Inmunologia Molecular. La Habana, Cuba. idania@cim.sld.cu. orcid.org/0000-0002-4478-4439

4 Instituto Finlay de Vacunas. La Habana, Cuba. malbojaire@finlay.edu.cu

- Autor de correspondencia: mjuliab@ind.cujae.edu.cu 
sector. The study is based on the use of the logical historical method, the modeling, as well as the documentary analysis, direct observation, and the expert method. In order to comply with the general objective, it was necessary to use the case study as a scientific research method, which allows investigating the human capital management of the High Technology Companies of the Cuban pharmaceutical biotechnology sector, in its real context and with all its complexities associated. The results lead to the establishment of a particular conceptual model that takes into account the singularities of a sector that is distinguished by the intensive use of knowledge and full-cycle companies. In addition, a general application procedure is shown, with specific work procedures, for which the best practices developed in different centers regarding human capital were taken into consideration.

Keywords: Competencies, Management, high technology enterprises

\section{INTRODUCCIÓN}

El desplazamiento del desarrollo económico hacia sectores intensivos en conocimiento, más conocido como economía basada en el conocimiento (Corredera, 2013; Eriksson, 2014; Ferreras-Méndez et al, 2015; Garriga, Von Krogh, \& Spaeth, 2013; Pérez, 2014), enmarca un tipo de empresa relativamente nueva en su incorporación al sector productivo, con un papel cada vez más importante en la generación de saberes, innovación y aprendizaje.

En Cuba, hay un llamado de la alta dirección del país para lograr que el sistema empresarial se integre por entidades eficientes, bien organizadas y eficaces, teniendo en cuenta que la baja eficiencia es uno de los factores que en el orden interno caracteriza la economía cubana.

En la política oficial vigente, en particular la Conceptualización del Modelo Económico y Social y el Plan de Desarrollo hacia el 2030 (Partido Comunista de Cuba, 2016), figura como necesidad de primer orden el incremento y la consolidación de los ingresos por concepto de exportaciones, así como el desarrollo de sectores estratégicos, entre los cuales se encuentra el biotecnológico farmacéutico. En este sector se integran empresas con impacto en la actividad económica del país y en la generación de empleos, además de una reconocida práctica tecnológica.

La industria biotecnológica farmacéutica representa la experiencia más convincente de empresas cubanas, que además de centros científicos, constituyen organizaciones económicas con resultados de impacto. La empresa de alta tecnología (EAT), innovadora, exportadora, económicamente viable, y propiedad del pueblo cubano, debe ser el actor principal de la articulación entre la ciencia y la economía (Lage, 2007).

La industria biotecnológica farmacéutica cubana y sus EAT tienen registros de sus productos, algunos de ellos únicos en el mundo, en más de 50 países de todos los continentes. Hoy es una rama estratégica para el desarrollo del país, no solo desde el punto de vista cuantitativo, sino también cualitativo. Los resultados productivos de BioCubaFarma contribuyen multidisciplinariamente al desarrollo de la economía nacional, pues el mayor potencial exportable de Cuba reside hoy en los artículos y los servicios de salud, cada vez más seguros, con alto valor agregado y destinados a la diversificación y el crecimiento constante. 
Esta industria, al mismo tiempo, es una de las primeras en el país que se ubica en el área de la llamada economía basada en el conocimiento. En este sentido es notorio que con cierre del año 2016 el sector en Cuba contaba con un total de 182 objetos de invención y 2336 patentes solicitadas, de ellas 543 concedidas en Cuba y 1816 en el exterior (Lage, 2016).

En el mundo contemporáneo globalizado, dinámico y cambiante, las empresas de alto desempeño como las del sector biotecnológico farmacéutico, han tenido que desarrollar nuevas tecnologías asociadas a la $\mathrm{GCH}$ (Gestión del Capital Humano) como proceso que permite a la empresa enfrentar los cambios y al mismo tiempo mantener su desarrollo y competencias en un mercado altamente competitivo (Albojaire \& López, 2015; Albojaire et al., 2014; Bella, 2013; Caballero, 2013; Cadalzo et al., 2016; Collins \& Clark, 2003; Chico, 2011; Dharmalingam, 2016; Ferreras-Méndez et al., 2015; Hillier-Fry, 2014; Huang \& Huarng, 2015).

Muchos autores se han encargado de articular lo que consideran más conveniente para gestionar el capital humano, sin embargo, no se agotan las exigencias para un sector caracterizado por la innovación, el uso intensivo del conocimiento y una fuerza de trabajo de alta calificación como factores decisivos de la productividad, así como por la complejidad de organizaciones que contienen dentro de sí el ciclo completo de vida útil de sus productos.

En este contexto, el presente artículo tiene como objetivo establecer algunos requerimientos que demanda la $\mathrm{GCH}$ en EAT del sector biotecnológico farmacéutico cubano y favorecer su enfoque estratégico.

En la literatura académica suelen emplearse diferentes denominaciones para referirse a los empleados, las más extendidas son recursos humanos (Corredera, 2013; Souto, 2015; Barba \& Serrano, 2015), talento humano (Chiavenato, 2011), capital humano (Hernández, 2011; Cuesta \& Valencia, 2014. Se pueden encontrar expresiones como gestión de personas y factor humano y la intención es destacar a quienes aportan con sus conocimientos, experiencias, habilidades y motivaciones a la generación de bienes.

Los estudios sobre el tema ofrecen representaciones y metodológicas que sugieren cómo atraer, desarrollar, comprometer y retener a los mejores profesionales. La GCH puede favorecer el desempeño organizacional de las empresas de alta tecnología si su diseño tiene un enfoque estratégico e integrador. Los progresos que promete el sector biotecnológico farmacéutico solo serán posibles si la $\mathrm{GCH}$ refleja en sus representaciones conceptuales el conocimiento como insumo limitante, la coexistencia de normas y regulaciones que exigen los diferentes sistemas de gestión, reconoce los múltiples riesgos laborales, entre otros desafíos importantes.

Por otra parte, los permanentes sistemas de monitoreo y control del comportamiento de todos estos procesos en el sector biotecnológico farmacéutico, evidencian que existen limitaciones relacionadas con la integración de sistemas de gestión, el enfoque a procesos y el enfoque de competencias aplicado a procesos estratégicos para el sector como la selección, la formación, la evaluación del desempeño y la retribución laboral.

La gran diversidad de sistemas de gestión (Organización General, Atención al Hombre, Organización de la Producción de Bienes y Servicios, Gestión de la Calidad, Planificación, Contratación Económica, Contabilidad, Control Interno, Relaciones Financieras, Costos, Precios, Informativo, Mercadotecnia, Capital Humano, Gestión Ambiental, Gestión de la 
Innovación, Comunicación Empresarial) (Consejo de Ministros, 2014; NC 3000:2007), cada uno con sus múltiples requisitos y exigencias, unido a la complejidad de los procesos propios biotecnológicos, que forman parte de la organización de ciclo cerrado y de alta tecnología, así como la gran variedad de responsabilidades comunes a cumplir y garantizar para las agencias regulatorias, convierten en demandas ineludibles, la gestión por procesos, la integración y el enfoque por competencias dentro de la $\mathrm{GCH}$.

La utilización de calificadores de cargos muy generales e importados de otros sectores o ramas, así como deficiencias alrededor de la elaboración y actualización de los Perfiles de Puestos de Trabajo, constituyen también oportunidades de mejora para la GCH en el sector. La ausencia de estudios que expliquen cómo gestionar el Capital Humano en las EAT del sector biotecnológico farmacéutico, limitan el desarrollo estratégico de la misma y en consecuencia las posibilidades de proyectarla en base a sus regularidades y rasgos distintivos.

Los modelos para la gestión integrada de capital humano (ONN, 2007; Cuesta, 2010; Chiavenato, 2011; García, 2011), constituyen una referencia importante para las organizaciones empresariales cubanas, sin embargo, no es suficiente para una $\mathrm{GCH}$ asociada a los retos de incertidumbre y sostenibilidad de la innovación que exigen las EAT del sector biotecnológico farmacéutico. Si las EAT del sector biotecnológico se caracterizan por la innovación, responsabilidad social, apoyo en sistemas de información, altos estándares en I + D, gestión de riesgos; el modelo de GCH debe responder a estas exigencias.

\section{MATERIALES Y MÉTODOS}

Se utilizó el estudio de caso exploratorio, evaluativo y múltiple, como método de investigación científica que, mediante la aplicación de técnicas cuantitativas y cualitativas, permitió describir y analizar la GCH en las EAT del sector biotecnológico farmacéutico, en su contexto real y con todas sus complejidades asociadas. Es un estudio de caso múltiple porque el interés se centra en el análisis intensivo de varias empresas del sector biotecnológico farmacéutico: Instituto Finlay de Vacunas (IFV), Centro de Inmunología Molecular (CIM), Centro de Ingeniería Genética y Biotecnología (CIGB), Centro de Química Biomolecular (CQB).

Entre los métodos teóricos se utilizó el histórico- lógico; este permitió la determinación de tendencias con respecto a la $\mathrm{GCH}$ en el sector biotecnológico farmacéutico. Se empleó también, el método inductivo-deductivo para comprender las regularidades de la $\mathrm{GCH}$ en el sector de referencia y diseñar un modelo de gestión estratégica.

Se utilizó el análisis documental, la observación directa, el método de expertos. Se tomaron en consideración las mejores prácticas desarrolladas en EAT del sector biotecnológico farmacéutico en materia de capital humano; aquellos modos de hacer el proceso de selección, evaluación del desempeño y formación, entre otros; que han contribuido a los resultados probados de la industria biotecnológica cubana. Se realizó un análisis de las particularidades de la GCH en el sector como forma de contextualizar cualquier propuesta de diseño (Cadalzo, 2017). 
Se integraron herramientas con enfoque a proceso, que enfatizan el valor agregado de la fuerza productiva competente y la integración con otros sistemas de gestión. Con esa información registrada, se diseñó un modelo conceptual y un procedimiento general de trabajo a fin de contribuir a la gestión estratégica del capital humano (GECH) en estos centros.

\section{RESULTADOS Y DISCUSIÓN}

\section{Modelo de la GECH- Bio.}

Como resultado de analizar las concepciones teóricas más reconocidas y sistematizar las experiencias que por años exhiben las EAT del sector biotecnológico farmacéutico cubano, se obtiene un modelo conceptual, en lo adelante Modelo de la GECH-Bio que explica el accionar de la gestión del capital humano y que se expresa en un procedimiento general de trabajo y contiene varios procedimientos específicos. Ambos requerimientos, modelo y procedimiento general, aportan un modo de gestionar el capital humano en el sector biotecnológico farmacéutico, que parte de sus singularidades.

Entre las bases teóricas esenciales en las cuales se sustenta el diseño del modelo está la búsqueda de un Enfoque estratégico (Cuesta \& Valencia, 2014; Peñalver, 2014; Chiavenato, 2011) que parta de la planeación empresarial, la misión, la visión, las políticas y los objetivos, tanto a nivel organizacional como a nivel de proceso. Se integran acciones y se diseñan procesos estratégicos para la $\mathrm{GCH}$, como el de competencias laborales.

El modelo se sustenta en un Enfoque de orientación al cliente (ISO 9001, 2015) a fin de garantizar la seguridad, calidad y eficacia de los medicamentos; se toman en consideración las demandas de las agencias regulatorias cuyas leyes y normas se convierten en requisitos de entrada y en elementos para la mejora continua. También se consideran las legislaciones y resoluciones laborales vigentes que amparan las condiciones de trabajo, formas y sistemas de pagos, así como la formación y desarrollo continuo de los trabajadores.

Esta orientación al cliente conduce a considerar el Enfoque a proceso (Cordero, 2015) ya que se parte de reconocer la GCH como proceso de la organización. Se utiliza la ficha de proceso como herramienta fundamental que contiene elementos de entrada y salida, así como un conjunto de etapas y actividades interrelacionadas entre sí que transforman y mejoran los procesos, añadiéndoles un nuevo valor para la organización.

También guio el diseño del modelo y el procedimiento, el enfoque de sistema (Corredera, 2013; García, 2011), porque hay un reconocimiento a la integración entre los procesos de $\mathrm{GCH}$ y de este con otros sistemas de gestión. Esta integración parte de la existencia de requisitos comunes entre estos elementos. Además, se toma a la gestión por competencias (Barroso, 2016; Cadalzo et al, 2016) como eje central de funcionamiento y al perfil del cargo como herramienta básica para la gestión de los puestos.

Constituyen condiciones básicas para la efectividad del modelo de la GECH-Bio las siguientes: Que exista la voluntad de la alta gerencia de alinear la estrategia de la organización con la 
de capital humano, que se identifiquen los procesos y se reconozca a la GCH como un proceso estratégico para la organización y que existan gestores de capital humano con las competencias requeridas. De no existir estas premisas, será necesario crearlas pues de lo contrario se compromete la efectividad del modelo y el procedimiento diseñados.

Se establecieron algunas normas que distinguen el modo de hacer la $\mathrm{GCH}$ en el sector biotecnológico farmacéutico y que sirven de principios para la ejecución de acciones como parte del procedimiento general y los específicos; ellos reflejan los núcleos teóricos que se tomaron como referencia:

1. Responsabilidad social de la organización con el medio ambiente y con todo el universo de clientes, en particular los pacientes y sus trabajadores.

2. La integración como demanda de todos los procesos organizacionales, a través de las fichas de procesos diseñadas y el perfil de cargo por competencias como herramientas integradoras.

3. La adaptabilidad del modelo para aplicarse en las empresas del sector biotecnológico farmacéutico, tomando en consideración el macro y el microentorno específico de las entidades.

4. La utilización de un enfoque de mejoramiento continuo de los procesos y del análisis de riesgo.

\section{La innovación como elemento distintivo del sector.}

La figura 1 es una representación gráfica del Modelo de la GECH- Bio. Como se aprecia, los elementos de entrada son:

- Legislaciones nacionales y extranjeras asociadas al sector empresarial en general y que el farmacéutico biotecnológico también debe cumplir.

- Convenciones, normas, guías y recursos de información que requiere el sector, como publicaciones, patentes, monografías, comportamiento del mercado farmacéutico, perfiles de empresas e instituciones académicas.

- Regulaciones farmacéuticas nacionales y extranjeras. El sector debe cumplir con un extenso marco regulatorio, en este sentido se ve obligado a vencer las fuertes y crecientes barreras no arancelarias que establecen las agencias regulatorias y que significan la posibilidad o no, de la entrada de los productos a nuevos territorios. El hecho de no considerar por ende estas regulaciones, puede significar para el sector, la no penetración de nuevos mercados importantes para el crecimiento y la sostenibilidad económica del mismo.

- Proyección estratégica y regulaciones de BioCubaFarma como entidad de control y orientación, así como facilitador de las relaciones de la empresa con otros organismos rectores y socios. 


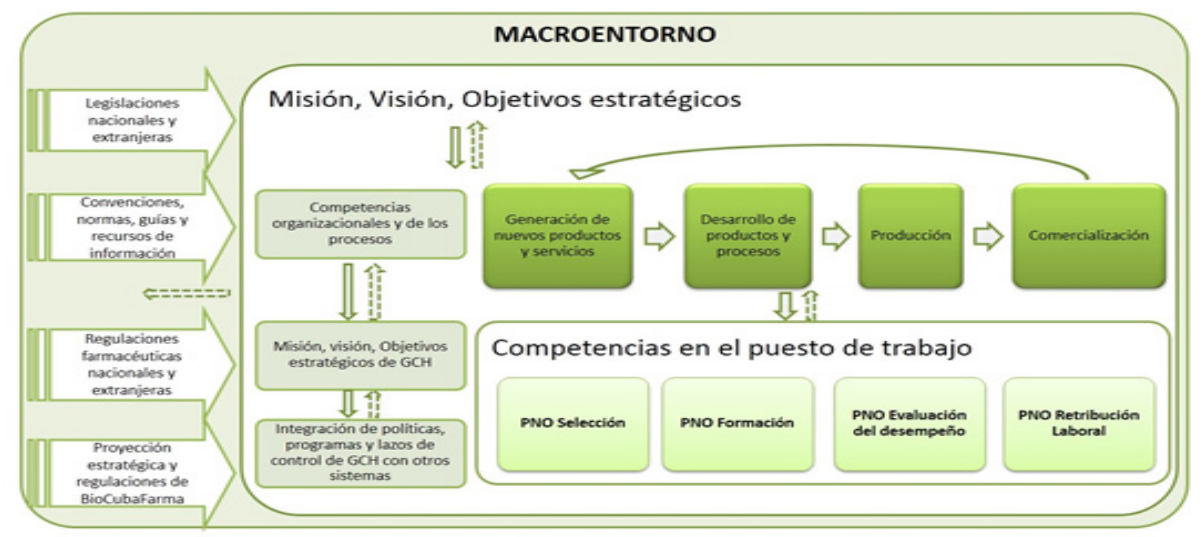

Figura 1. Representación gráfica del Modelo GECH- Bio

Estas entradas representan el vínculo con el contexto de barreras arancelarias y no arancelarias, en el que se mueve la organización para poder diversificar sus productos, mercados y establecer sus modelos de negocios; con esos elementos se conforma la estrategia empresarial para la $\mathrm{GCH}$. Muestran lo relevante del contexto (internacional y nacional) y facilitan la coordinación y el control de los elementos del sistema.

Los elementos de salida son los procesos de capital humano diseñados: competencias laborales, selección e integración, formación y desarrollo, evaluación del desempeño, retribución laboral; considerados todos como estratégicos para la $\mathrm{GCH}$ en el sector.

Entre estos elementos hay flechas que indican interrelación e intercambio permanente entre los procesos, las competencias organizacionales y la proyección estratégica de la organización. Las saetas más grandes y continuas muestran entradas de obligatorio cumplimiento, y las discontinuas y hacia arriba, indican una retroalimentación dada por los resultados que se van obteniendo como consecuencia de la propia aplicación. Las flechas de doble saeta indican regulación para la mejora continua entre los componentes del modelo.

Los objetivos estratégicos de la empresa se adecuan al entorno internacional, al del país y a su objeto social. Esto demuestra el enfoque de riesgo y mejora continua que permite trabajar a la empresa potenciando sus fortalezas y mitigando o eliminando sus debilidades. La organización requiere políticas y procedimientos de inteligencia empresarial para anticiparse a través del uso de diferentes herramientas a las amenazas oportunidades del entorno. Se acompaña de estudios de escenarios y riesgos para el logro de los objetivos estratégicos y la gestión por competencias de su capital humano. Se consideran las competencias organizacionales que representan aquellos comportamientos distintivos de la entidad.

Las políticas empresariales deben aplicarse a todos los procesos y documentarse en reglamentos, manuales, expedientes de control interno donde se plasman los riesgos al cumplimiento de los objetivos empresariales a corto, mediano y largo plazo. Deben fomentar que se seleccionen, formen, evalúen y retribuyan a los trabajadores con el propósito de que tengan las competencias requeridas para trabajar en una empresa biofarmacéutica de alta tecnología, con capacidad innovadora para generar bienes y 
servicios, con impacto en la economía y la salud poblacional. Los lazos de control se expresan a través de la medición de las no conformidades cuya criticidad se establece en función de un análisis de riesgos y la mitigación de insatisfacciones a través de la utilización de un enfoque de competencias. La estrategia empresarial para la GCH se nutre de la diseñada para la empresa. Esta a su vez contiene misión, visión y objetivos estratégicos.

La gestión por competencias como enfoque guían la estrategia empresarial para la GCH en los tres niveles convencionalmente reconocidos: organización, procesos, puestos de trabajo. Las competencias organizacionales que aprueben guiarán la elaboración y actualización de los reglamentos, manuales, expedientes de control interno, en donde se plasman los riesgos a los objetivos empresariales a corto, mediano y largo plazo, así como el funcionamiento de los sistemas de gestión.

La GCH a través de procedimientos de planificación, ejecución y control de los objetivos garantiza el cumplimiento de las competencias que requieren los procesos de generación, desarrollo, producción y comercialización de productos.

Los procesos estratégicos de la GCH: selección e integración, formación y desarrollo, evaluación del desempeño y retribución laboral se gestionan tomando como referencia las competencias reflejadas en los perfiles de puestos de trabajo. Estos procesos se describen, documentan y aprueban a través de PNO (procedimientos normalizados de operaciones) que forman parte del sistema de documentación perteneciente al SGC (sistema de gestión de la calidad). De esta manera se van alineando las políticas del macroentorno con el microentorno empresarial y el puesto de trabajo.

Este modelo constituye el patrón de información para la implementación del procedimiento. La existencia de entradas, salidas, actividades debe verse complementada con otros elementos que aporta el enfoque de Arquitectura Empresarial (Ortega, 2016) y que en esta propuesta no se tuvieron en cuenta; es el caso de roles, eventos, flujo informativo, lo que contribuiría no solo a describir sino a explicar las interrelaciones y con carácter mediato automatizar los procesos.

\section{Procedimiento general de trabajo.}

Se diseñó un procedimiento general de trabajo, con procedimientos específicos asociados, el cual se muestra en la figura 2. El procedimiento general comprende cinco etapas. En la primera se determinan las regularidades de la $\mathrm{GCH}$ mediante un diagnóstico de la situación actual de la misma. En la segunda etapa se diseñan los procesos que se consideran estratégicos para la GCH en el sector biotecnológico farmacéutico, con sus procedimientos específicos de trabajo asociados. El diseño se realizó a través de fichas técnicas que contienen: diagramas de flujo, descripción del procedimiento específico de trabajo, mapas de riesgos, indicadores, competencias. Los procedimientos específicos que se obtienen se convirtieron en 14 PNO del Sistema de Documentación de la Dirección de Calidad con 33 registros propios, que le dan mayor coherencia y aplicabilidad al procedimiento general de trabajo.

La tercera etapa se orienta a la implementación de los procesos diseñados. En la cuarta se miden, controlan y analizan los resultados; para lo cual se utilizan indicadores y un enfoque de análisis de riesgos asociados a la detección de no conformidades, la determinación de su criticidad y a la elaboración de acciones correctivas y preventivas para la solución de los problemas. La quinta etapa orienta la planificación y aplicación de las mejoras que permitirán alcanzar un nivel superior en el desarrollo cíclico de la $\mathrm{GECH}$. Una versión detallada del procedimiento es posible consultarla en Cadalzo (2017) 


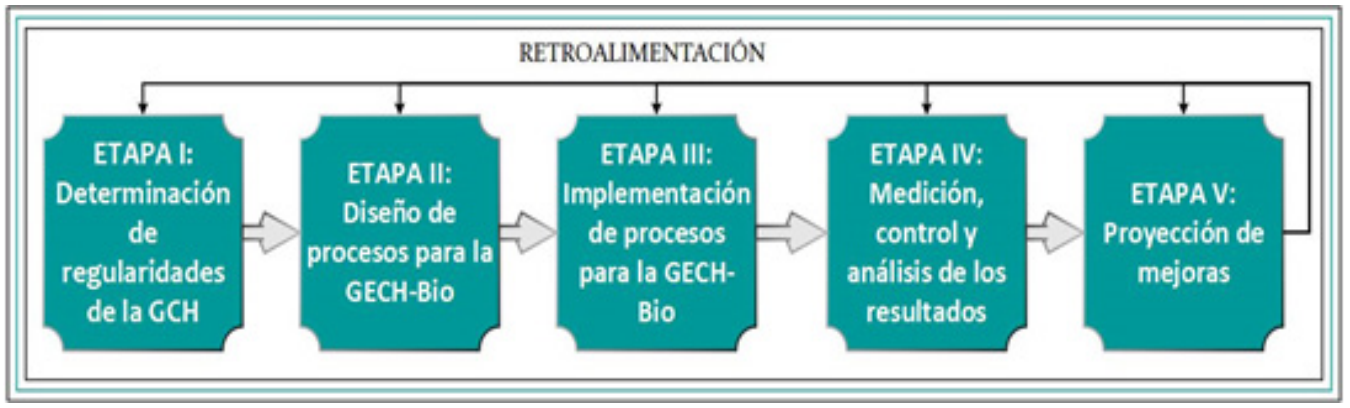

Figura 2. Procedimiento general de trabajo para la GECH-Bio

El procedimiento general de trabajo y los procedimientos específicos de operación que éste contiene garantizan un modo de hacer organizado para la GECH en el sector objeto de estudio, lo que representa una notable contribución al desarrollo económico y social, si se tiene en cuenta que la alta dirección del país ha hecho un llamado a lograr que el sistema empresarial se integre por entidades eficientes, bien organizadas y eficaces.

Por su diseño, tanto el modelo como el procedimiento general de trabajo, responde a las exigencias de las Agencias Regulatorias en materia de capital humano, contribuyen a la integración del sistema de gestión de capital humano (SGCH) hacia lo interno y con otros sistemas de trabajo inherentes a este tipo de empresas y permite el mejoramiento continuo para alcanzar mayores niveles de efectividad. Contribuyen a que las empresas del sector obtengan, en materia de $\mathrm{GCH}$, la condición de "Cumplimiento de BPF" otorgada por las diversas agencias regulatorias nacionales y extranjeras, requisito este indispensable para la comercialización de los productos de la industria biotecnológica y continuar obteniendo y/o renovando las licencias de la producción y exportación de los mismos.

\section{Validación del modelo y el procedimiento general de trabajo.}

Se realizó una validación teórica y otra por la constatación de cambios en indicadores de impacto. Para la validación teórica se comparó el modelo propuesto con 62 referentes que abordaban la GCH (Cadalzo, 2017). Las fuentes bibliográficas se compararon con respecto a los supuestos teóricos que se convirtieron en requisitos de diseño: enfoque estratégico, de procesos, de competencias, enfoque integrador, investigación-desarrollo-innovación, contextualizado al sector y perfil de cargo.

El $76 \%$ de los referentes analizados pertenece a los últimos cinco años y recogen además experiencias de países bien posicionados en el sector biotecnológico, en su mayoría, Cuba, Estados Unidos y la Región Europea. Cada referente fue evaluado con criterios de 1, 3 y 5; significando el valor 1 que no aparece el enfoque, el 3 que aparece o se logra solo parcialmente y el valor 5 que está completamente logrado. El estadígrafo utilizado para la validación teórica fue la moda, ampliamente utilizado en este tipo de estudio. 


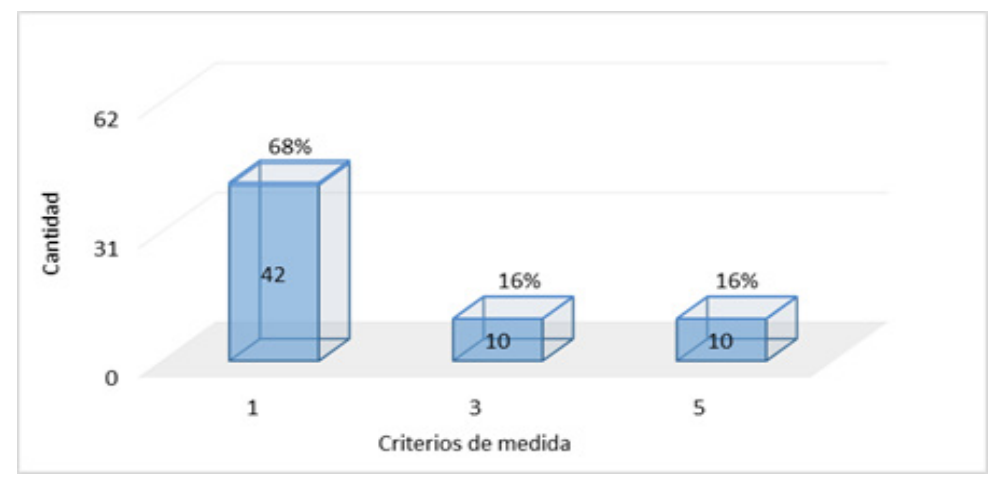

Figura 3. Resultados de la validación teórica

En la figura 3 se puede observar que 42 referentes toman valor 1, lo que significa que el $68 \%$ (más de la mitad de la muestra analizada) no contiene dentro de sí las variables de comparación, 10 de ellos (el 16\%) solo las abarca parcialmente y otro $16 \%$ obtuvo una moda de 5 puntos, porcentaje bajo en este contexto de análisis.

El uso del procedimiento permitió observar avances en las empresas estudiadas (Cadalzo, 2017). Se detallan los resultados en el Centro de Inmunología Molecular con respecto a la determinación y aprobación del sistema de competencias laborales, en los procesos de formación y desarrollo, retribución laboral e indicadores de empleo y salario.

\section{Competencias laborales}

Antes del 2014 no se utilizó ese enfoque de forma explícita en la GCH.

2014 - Las competencias organizacionales y de procesos se aprobaron en una versión primera. Como resultado de la investigación y aplicación se realizó la defensa de una tesis de maestría. Se conformó el Comité de Competencias del centro.

2015 - Se aplicaron en el centro herramientas establecidas para la conceptualización de las competencias organizacionales y se aprobaron las mismas. Se revisaron y actualizaron las competencias de procesos hasta ese entonces definidas. Mejoramiento del Comité de Competencias creado, dándose al mismo un enfoque más integral en su funcionamiento. Creación de un procedimiento para el trabajo en general con las competencias en la organización.

2016 - Actualización y aprobación de las competencias de proceso y comienzo de la definición y aprobación de las competencias a nivel de puestos de trabajo. Se comienza a modificar el plan de formación, apareciendo el enfoque de los planes de formación por procesos, de tal manera que estos contribuyan de una mejor manera a las competencias de los procesos y de los cargos. Se asigna para la atención técnico metodológica de esos planes de formación por procesos a una Facultad determinada de los diferentes centros de la Educación Superior. Se determinan las competencias del proceso de $\mathrm{CH}$ y del gestor de ese proceso. Defensa de una tesis de maestría.

\section{Formación y Desarrollo del personal}

Se muestran indicadores relacionados con este proceso en la tabla 1. 
Tabla 1. Indicadores del proceso de Formación y Desarrollo en el CIM

\begin{tabular}{|l|c|c|c|c|}
\hline \multicolumn{1}{|c|}{ Indicador } & $\mathbf{2 0 1 2}$ & $\mathbf{2 0 1 3}$ & $\mathbf{2 0 1 4}$ & $\mathbf{2 0 1 5}$ \\
\hline Cantidad de trabajadores anualmente formados en la empresa & 1253 & 1284 & 1646 & 2369 \\
\hline Cantidad de acciones de formación internas realizadas en el año & 35 & 45 & 47 & 74 \\
\hline Porciento de cumplimiento del Plan de Formación Institucional & $85 \%$ & $87 \%$ & $88 \%$ & $100 \%$ \\
\hline Cantidad de personal con título académico y grados científicos & 159 & 166 & 197 & 201 \\
\hline
\end{tabular}

\section{Retribución Laboral}

Se muestran indicadores relacionados con este proceso en la tabla 2. Los indicadores anteriores reflejan como tendencia un comportamiento en ascenso y positivo a lo largo de los años, lo cual valida de forma retrospectiva el procedimiento diseñado.

Tabla 2. Indicadores del proceso de Retribución Laboral en el CIM

\begin{tabular}{|c|r|r|r|r|}
\hline Indicador & $\mathbf{2 0 1 2}$ & $\mathbf{2 0 1 3}$ & $\mathbf{2 0 1 4}$ & $\mathbf{2 0 1 5}$ \\
\hline VAB(MMUM) & 201,00 & 189,151 & 177,672 & 183,530 \\
\hline Salario medio(CUP) & 1600 & 1418 & 1937 & 2838 \\
\hline $\begin{array}{c}\text { Productividad } \\
\text { (CUP/Trabajador) }\end{array}$ & 196480 & 174332 & 163509 & 178664 \\
\hline
\end{tabular}

Con una mejor $\mathrm{GCH}$ que labora en el sector biotecnológico farmacéutico, se contribuye a elevar la calidad de vida de los pacientes y erradicar o mitigar el efecto de las enfermedades en Cuba y el exterior. Si se asume que el capital humano influye en el desempeño de las EAT, en tanto es el portador del conocimiento que genera los altos niveles de productividad, se estará de acuerdo en que esta efectividad puede incrementarse en la medida que se logre su gestión estratégica.

Por primera vez se integra en una propuesta la interrelación entre los procesos que tributan a la GECH como lo demanda este tipo de organización, que por el poco tiempo del sector en Cuba y el mundo, comienza ahora a conceptualizarse y determinar sus regularidades y diferencias con respecto a otros sectores menos intensivos en el uso del conocimiento.

La utilización del procedimiento como concreción de un modelo conceptual contribuye a que el personal trabaje con la calidad requerida y a minimizar la introducción de cambios no previstos en los procesos de trabajo. Posibilita la adecuada calificación, mejoramiento continuo y el desarrollo del personal involucrado en todos los procesos que comprenden el ciclo completo (investigación, desarrollo, producción y comercialización) de una organización que se encarga de la prevención o disminución de las enfermedades, tanto para la población cubana como la de otras regiones del mundo. 


\section{CONCLUSIONES}

Este trabajo se enfocó en mostrar que las empresas del sector biotecnológico farmacéutico cubano, requieren de un modelo conceptual que visibilice la pertinencia de la GECH y un procedimiento general de trabajo, con procedimientos específicos que permitan su perfeccionamiento. Si las EAT del sector biotecnológico farmacéutico demandan innovación, responsabilidad social, apoyo en sistemas de información, altos estándares en Investigación y Desarrollo, gestión de riesgos, la GCH debe responder a estas exigencias con el diseño de sus procesos estratégicos.

Para el diseño del modelo y el procedimiento general de trabajo se consideraron las ventajas y limitaciones de las propuestas existentes, el marco regulatorio y legislativo, las tendencias actuales en la $\mathrm{GCH}$ y las regularidades específicas del sector en el contexto cubano; estas últimas propician un mayor conocimiento del sistema y la posibilidad de una mayor preparación para la variabilidad de los procesos asociados a las personas y mejor toma de decisión ante las contingencias.

El modelo y el procedimiento general de trabajo se validaron mediante la comparación con fuentes teóricas, y el cumplimiento de indicadores de impacto. Los resultados satisfactorios permiten inferir que contribuyen al desarrollo de la GECH.

\section{AGRADECIMIENTOS}

Los autores desean agradecer al colectivo de profesores de la Facultad, en particular a los integrantes del proyecto de investigación "Gestión estratégica integrada de recursos humanos con enfoque de competencias en organizaciones laborales", en el cual se insertan estos resultados. También al Departamento de Ingeniería Industrial de la Universidad Tecnológica de La Habana (CUJAE), así como a todos aquellos técnicos y especialistas del Instituto Finlay de Vacunas y de otros centros de BioCubaFarma, que contribuyeron a los resultados obtenidos.

\section{REFERENCIAS}

ALBOJAIRE, M; LÓPEZ R. Diseño y aplicación de un procedimiento para la determinación del sistema de competencias en el Instituto Finlay [en línea]. Trabajo de Diploma. Instituto Superior Politécnico José Antonio Echeverría, La Habana, Cuba, 2015. Disponible en: http:// hdl.handle.net/123456789/1867

ALBOJAIRE, M; LOPEZ, R. y LARRAINAGA, L. "Diagnóstico de la Gestión de los Recursos Humanos en Empresas de Alta Tecnología." Instituto Finlay - Departamento de Recursos Humanos, pp. 10, 2014.

BARROSO, Y. M. Aplicación del enfoque por competencias laborales en la sucursal la 
habana de ARTEX, S.A [en línea]. Tesis de Maestría. Instituto Superior Politécnico José Antonio Echeverría, La Habana, Cuba, 2016. Disponible en: http://tesis.cujae.edu.cu:8080/ xmlui/handle/123456789/3456

BELLA, B. Evaluación del modelo de Entrenamiento en Buenas Prácticas del CIM con relación a las exigencias de las agencias regulatorias biofarmacéuticas internacionales. Tesis Master. Universidad de la Habana. Instituto de Economía, Ciudad de la Habana, 2013.

CABALLERO, I. "La historia del CIM contada por sus trabajadores" Centro de Inmunología Molecular, La Habana. 2013.

CADALZO, Y; BECERRRA, MJ; ALBOJAIRE, M. y LOPEZ, R. (2016). Determinación de las competencias organizacionales y de procesos en un centro del sector biofarmacéutico Vaccimonitor, vol. 25, n. 3, pp. 77-83.

CADALZO, Y. Tecnología para la gestión del capital humano en empresas de alta tecnología del sector biotecnológico farmacéutico [en línea]. Tesis de Doctorado. Universidad Tecnológica de La Habana José Antonio Echeverría, Cuba, 2017. Disponible en: http://tesis.cujae.edu. cu:8080/handle/123456789/9105

CHIAVENATO, I. Administración de los Recursos Humanos. 9 edición. New York: Mc Graw Hill, 2015. ISBN 978-968-451-433-1.

CHICO, E. Propuesta de tratamiento económico para las organizaciones de la Biotecnología Cubana. Tesis de Diplomado. Universidad de la Habana. Facultad de Economía. La Habana, 2011.

COLLINS, C. y CLARK, K. (2003). Strategic human resource practices, top management team social networks, and firm performance: The role of human resource practices in creating organizational competitive advantage Academy of management Journal, v. 46, n.6, 740-751.

CONSEJO DE MINISTROS. Decreto Ley 252 y Decreto 281 actualizados y ampliados. La Habana: Ministerio de Justicia, 2014.

CORDERO, J. P. La Gestión por Procesos en el Instituto Finlay. Análisis y Mejora del Proceso de Distribución y Ventas. [en línea]. Trabajo de Diploma, Universidad de La Habana, La Habana. 2015. [consultado: Disponible en: http://dspace.uh.cu:8080/xmlui/handle/123456789/2896

CORREDERA, E. Gestión de los recursos humanos en empresas innovadoras. Tesis de Maestría, Universidad del País Vasco, España, 2013.

CUESTA, A. Tecnología de Gestión de Recursos Humanos. 3 edición. La Habana: Editorial "Félix Varela". 2010. ISBN 978-959-07-1340-8 OC.

CUESTA, A. y Valencia, M. Indicadores de Gestión de CH y el Conocimiento en la empresa. Bogotá: ECOE Ediciones .2014.

DHARMALINGAM, K. (2016). Human resource development in biotechnology. Current Science, v.110, n. 4, 1-2.

ERIKSSON, T. (2014). Processes, antecedents and outcomes of dynamic capabilities". Scandinavian Journal of Management, v.30, n.1, 65-82.

FERRERAS-MÉNDEZ, J. L., Newell, S., Fernández-Mesa, A. y Alegre, J. (2015). Depth and breadth of external knowledge search and performance: The mediating role of absorptive capacity. Industrial Marketing Management, n. 47, 86-97. 
GARCÍA, V. Procedimiento para la implementación de la Gestión del Capital Humano en servicios asistenciales de hospitales [en línea]. Tesis de Doctorado. Instituto Superior Politécnico José Antonio Echeverría, La Habana, Cuba, 2011. Disponible en: http://hdl. handle.net/123456789/1277

GARRIGA, H.; VON KROGH, G. y SPAETH S. (2013). How constraints and knowledge impact open innovation". Strategic Management Journal, v.34, n.9, 134-1144

HERNÁNDEZ, I. Diseño y aplicación de una Tecnología de Aprendizaje Organizacional para la implementación del Sistema de Gestión Integrada de Capital Humano. Casos de estudio: Empresas del Grupo Empresarial de la Construcción de Pinar del Río y de la Red de Capital Humano [en línea]. Tesis de Doctorado. Instituto Superior Politécnico "José Antonio Echeverría", La Habana, Cuba, 2011. Disponible en: http://tesis.cujae.edu.cu:8080/xmlui/ handle/123456789/6230

HILLIER-FRY, C. (2014). Recursos Humanos, agente del cambio en la industria farmacéutica. pharma-market.es [en línea]., 65-72. Disponible en : https://www.peoplematters.com/ Archivos/Descargas/Docs/Docs/articulos/1402_PharmaMarket.pdf

HUANG, C y HUARNG, K.H. (2015). Evaluating the performance of biotechnology companies by causal recipes. Journal of Business Research, v.68, n.4, 851-856.

ISO 9001. Quality management systems -- Requirements. Geneva: International Organization for Standardization, 2015.

LAGE, A. (2007). Conectando la Ciencia a la Economía: Las palancas del Socialismo. Revista Cuba Socialista, v. 45, 2-28. Cuba. ISNN 0864-0424.

LAGE, A. El contexto mundial de la industria biofarmacéutica: Desafíos y oportunidades para BIOCUBAFARMA" En: Congreso Internacional IDIFARMA'2016, Cuba, La Habana, 2016.

OFICINA NACIONAL DE NORMALIZACIÓN. Sistema de Gestión Integrado de Capital Humano - Vocabulario. NC 3000:2007. La Habana-Cuba: Oficina Nacional de Normalización, 2007.

ORTEGA, Y. Modelo de sistematización del conocimiento ontológico para la integración de tecnologías de información en el contexto organizacional [en línea]. Tesis de Doctorado. Universidad Tecnológica de La Habana José Antonio Echeverría, Cuba, 2016. Disponible en: http://tesis.cujae.edu.cu:8080/xmlui/handle/123456789/7716

PARTIDO COMUNISTA DE CUBA. Conceptualización del modelo económico y social cubano de desarrollo socialista, La Habana, Ministerio de Justicia, 2016.

PÉREZ, M. Capacidad dinámica de aprendizaje organizacional en la empresa de alta tecnología del sector biotecnológico cubano [en línea]. Tesis de doctorado, Universidad Central "Marta Abreu", Santa Clara, Cuba, 2014. Disponible en: http://tesis.cujae.edu. cu:8080/xmlui/handle/123456789/6324

PEÑALVER, A. Las tendencias de Recursos Humanos que veremos en 2014 [En línea]. Disponible en: http://antoniopenalver.blogspot.com/2014/01/las-tendencias-de-recursoshumanos-que.html.

SOUTO, L. Modelo de Gestión de Recursos Humanos para la Organización Superior de Dirección Empresarial del Ministerio de Comercio Exterior de Cuba. Tesis de Doctorado, Universidad de la Habana, 2015. 


\section{BIOGRAFIA}

Yanela Cadalzo Díaz, Ing. Industrial, Doctora en Ciencias Técnicas, Tecnólogo de Primer Nivel de la Dirección de Capital Humano del Instituto Finlay de Vacunas.

María Julia Becerra Alonso, Lic. en Psicología, Profesora Titular, Doctora en Ciencias Pedagógicas, Vicedecana de Extensión Universitaria, Facultad de Ing. Industrial, CUJAE.

Idania Caballero Torres, Investigadora y Profesora Titular, Doctora en Ciencias Biológicas, Tecnólogo de Primer Nivel de la Dirección de Capital Humano del Centro de Inmunología Molecular.

Mirelis Albojaire Santamaría, Ing. Industrial, Especialista de la Dirección de Capital Humano del Instituto Finlay de Vacunas. 